Review

\title{
Stressed brain, diseased heart: A review on the pathophysiologic mechanisms of neurocardiology
}

\author{
Vitor Hugo Pereira ${ }^{\text {a,b,c }}$, João José Cerqueira ${ }^{\text {a,b }}$, Joana Almeida Palha ${ }^{\text {a,b }}$, Nuno Sousa ${ }^{\text {a,b,* }}$ \\ a Life and Health Sciences Research Institute (ICVS), School of Health Sciences, University of Minho, Braga, Portugal \\ b ICVS/3B's, PT Government Associate Laboratory, Braga/Guimarães, Portugal \\ c Cardiology Department, Centro Hospitalar do Alto Ave, Guimarães, Portugal
}

\section{A R T I C L E I N F O}

\section{Article history:}

Received 3 November 2011

Received in revised form 23 January 2012

Accepted 25 March 2012

Available online $\mathrm{xxxx}$

\section{Keywords:}

Cardiovascular diseases

Central nervous system

Neurocardiology

Pathophysiology

Chronic stress

\begin{abstract}
A B S T R A C T
Cardiovascular diseases are traditionally related to well known risk factors like dyslipidemia, smoking, diabetes and hypertension. More recently, stress, anxiety and depression have been proposed as risk factors for cardiovascular diseases including heart failure, ischemic disease, hypertension and arrhythmias. Interestingly, this association has been established largely on the basis of epidemiological data, due to insufficient knowledge on the underlying pathophysiologic mechanisms. This review will revisit evidence on the interaction between the cardiovascular and nervous systems, highlighting the perspective on how the central nervous system is involved in the pathogenesis of cardiovascular diseases. Such knowledge is likely to be of relevance for the development of better strategies to treat patients in a holistic perspective.
\end{abstract}

(c) 2012 Elsevier Ireland Ltd. All rights reserved.

\section{Introduction}

Cardiovascular diseases (CVD) are the leading cause of death worldwide, being responsible for almost $32 \%$ of all deaths in women and $27 \%$ in men in 2004 and expected to kill 23.4 million people by 2030 if current trends remain [1]. While elevated blood levels of cholesterol, hypertension, diabetes mellitus and smoking are well-known riskfactors for CVD [2], understanding how other factors contribute to this burden is essential to develop new strategies to combat and/or prevent it. Among these, the central nervous system (CNS), in particular the stress response seems to be of relevance in the pathogenesis of CVD.

This review focuses on neurocardiology, highlighting the effects of central circuits over the control of cardiovascular system and on how peripheral mediators acting on specific brain regions influence neurocardiac conditions.

\section{Neurocardiology: the facts}

Psychiatric and neurologic diseases are positively associated with CVD. Epidemiological data clearly suggests that depression is an independent risk factor for myocardial infarction (AMI) and heart diseases in general $[3,4]$. Prospective studies with depressed individuals showed that a history of a major depressive episode was associated with

\footnotetext{
* Corresponding author at: Life and Health Sciences Research Institute (ICVS), Campus de Gualtar, University of Minho, 4710-057 Braga, Portugal. Tel.: + 351253 604806.

E-mail address: njcsousa@ecsaude.uminho.pt (N. Sousa).
}

a higher risk of AMI, even after correction for major coronary risk factors [5-7]. Of interest, the same was observed for both men and women [7]. In fact, a meta-analysis of 11 longitudinal studies revealed that depression is associated with a 1.64 relative risk (ranging from 1.50 to 4.16 in original studies) for development of coronary heart disease (CHD) [8]. Unfortunately, most of the studies measured depression at a single time-point during the follow-up period, which precludes the analysis on how time of exposure to depression was associated with CHD.

The impact of depression is also important in patients with already established CHD. Long-term mortality was higher (cardiac and all-causes) in patients who presented depression on hospital admission and/or on follow up $[9,10]$. This outcome persisted, although less powerfully, after correction for socio-demographic and biobehavioral characteristics, use of antidepressants and CVD medications [10]. Interestingly, patients only with depression displayed a higher risk of mortality (OR 2.10) than those only with CHD (OR 1.67) while those with both conditions displayed an additive risk of mortality (OR 4.99) reinforcing the importance of affective disorders on CVD [10]. The consequence of depression on heart diseases extends to other conditions including sudden cardiac death $[11,12]$ and heart failure (HF) [13,14]. Neurologic conditions are also implicated in the pathophysiology of several cardiac conditions: e.g. both acute stroke and epilepsy are associated with sudden death and de novo ECG alterations [15-18].

\section{The relevance of stress in neurocardiology}

Stress is a state of threatened homeostasis. For re-establishment of the equilibrium a repertoire of physiologic and behavioral responses 
is rapidly mobilized, constituting the adaptive stress response [19]. The adverse consequences of stress result from the inability of the individual to cope with the stressful stimuli or from maladaptive responses that may restore the homeostasis in short-term but may impose damage at different body systems in the long-term. It is a common belief that daily stressful situations predispose individuals to adverse cardiovascular events. The first reports of cardiac deaths related with stress date from 1942 Cannon's paper "voodoo death" in which the author suggested that an extreme condition of frighten inflicted to the victim might explain the multiple cases of tribal voodoo death [20]. Acute stress, elicited by natural disasters like earthquakes is likewise related with an increase in cardiac events and sudden death [21].

Takotsubo cardiomyopathy (or "stress cardiomyopathy") is probably the most remarkable example of how stress promotes direct heart injuries and will be discussed in further sections. More recently, the INTERHEART study investigated the associations of several psychosocial stressors with the risk of AMI [22]. This study compared 11,119 patients with a previous event of AMI with 13,648 patients free of clinical heart disease. Psychosocial stress was assessed by four simple questions about stress at work and at home, financial stress, and major life events in the past year; results estimated a 1.38 fold greater risk of AMI for patients who went through several periods of work stress and 2.14 for those who experienced periods of permanent stress at work [22].

In laboratory settings, the effects of chronic stress have been widely studied in rodents mainly using the chronic mild stress (CMS) model in which animals are exposed during 4 to 6 weeks to an unpredictable sequence of mild stressors [23]. CMS rodents not only display depressive-like behavior but also increased anxiety and impaired cognition, highlighting the fact that the co-morbid effects of human depression can also be observed in rodents [24]. Of notice, these animals present other anomalies, namely elevated heart rate (HR), reduced HR variability (HRV), elevated sympathetic tone and exacerbated cardiovascular reactivity when submitted to external stressors, suggesting that stress promotes an autonomic imbalance in favor of sympathetic system $[25,26]$.

\section{Neuronal networks implicated in cardiovascular regulation: the effects of stress}

Cardiovascular regulation by CNS has been widely discussed in the literature. Cardiovascular function is regulated by the autonomic nervous system, which encompasses two major divisions: the sympathetic and the parasympathetic system; the appropriate balance (autonomic tone) between the two is fundamental to the pathophysiology of CVD.

The organization of the autonomic nervous system (Fig. 1) is complex (for a review see [27]). It is widely recognized that regulation of cardiac function is dependent on medullary centers, namely the nucleus of the solitary tract (NST) and the rostroventrolateral medulla (RVLM) [27,28]. While the former receives afferents from baroreceptors and the visceral sensorial information derived from cranial nerves (including the vagus), the RVLM is mainly composed by excitatory neurons that are responsible for the generation of the sympathetic response. Although these regions generate reflex responses that may orchestrate timing cardiac adaptations, it is now clear that cardiac regulation is also dependent on supramedullary regions.

The insular cortex (IC) is a critical area in controlling the parasympathetic and the sympathetic tones. Not surprisingly, middle cerebral artery stroke victims, with insular damage, are prone to cardiovascular sudden death and autonomic alterations [29]. Studies with synchronized electric stimulation in animal models uncovered a chronotropic map in the IC [30]. While pure tachycardia was produced by stimulation of the rostral posterior insula, bradycardia was produced from the caudal posterior insula. Interestingly, there is evidence for a lateralization of cardiac control in this brain region: while the sympathetic tone is

\section{Afferent Pathway $\quad$ Efferent Pathway}

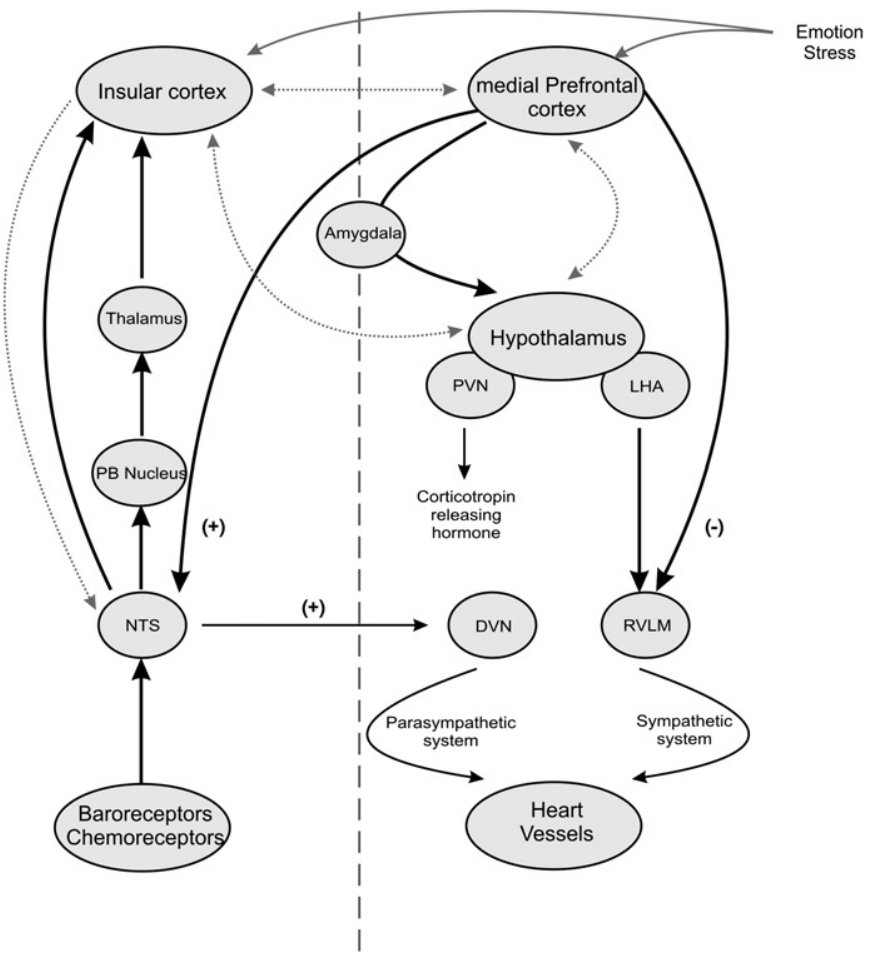

Fig. 1. Simplified schematic representation of the anatomical organization of autonomous nervous system in mammals. The regulation of sympathetic and parasympathetic systems involves a complex interaction between cortical and subcortical and medullary loci and peripheral organs. Apart from the more established efferent and afferent pathways (full lines), there are multiple interconnections between the different loci involved in the modulation of the autonomous nervous system (dot gray lines). In many cases their functional significance is still largely unknown but is likely to be relevant for the proper integration of the multiple inputs that control brain-periphery interactions. DVN-dorsal vagal nucleus; LHA-lateral hypothalamic area; NTS-nucleus of the solitary tract; PBparabrachial; PVN-paraventricular nucleus; RVLM-rostroventral lateral medulla.

predominantly regulated by right insular regions, parasympathetic cardiac manifestations are regulated by the left insula [31,32]. Clinical data collected in stroke patients showed that cardiac arrhythmia produced by unbalanced cardiac autonomic activity favoring the sympathetic system was more common after cerebral infarction on the right hemisphere [33]. Therefore the mechanism of cardiovascular instability following stroke seems to result from loss of the inhibition exerted by the right IC over inferior areas of cardiac control that predisposes to rhythm instability [34].

The involvement of the IC in stroke patients is easily documented by imaging methods; on the contrary, documenting a relation between stress and IC dysfunction, although important in light of the previous section, is not straightforward and is scarce [35]. Preliminary data in animals from our lab revealed that the IC is indeed a target of chronic stress (Pereira VH et al., unpublished observations).

The medial prefrontal cortex ( $\mathrm{mPFC}$ ) is a subdivision of the rodent's prefrontal cortex composed by several cortical areas (e.g. frontal area 2, dorsal and ventral anterior cingulate areas, prelimbic, infralimbic and medial orbital areas) [36]. Besides its implication in several cognitive functions [37] the mPFC is also involved in the regulation of cardiovascular functions [38,39]. The mPFC is a well-known target of stress. It has been shown that chronic stress leads to volume loss and dendritic atrophy of the MPFC; more prominently in the left hemisphere [36,37], which may, by decreasing left-to-right mPFC inhibition, cause an autonomic imbalance in favor of the sympathetic system. In this sense, the shift of a predominant parasympathetic tone in the normal functioning $\mathrm{mPFC}$ toward a predominant sympathetic tone in the "stressed" mPFC may be of major importance in the pathophysiology of 
stress-related CVD. This is corroborated by lesion studies in humans in which reduced parasympathetic modulation in patients with ventral mPFC lesions was suggested to increase the risk of anxiety, hypertension and arrhythmias [40].

The hypothalamus, namely through posterolateral hypothalamus (PLH) and paraventricular nucleus (PVN), is also a crucial center of integration of different visceral stimulus $[41,42]$. The PVN was shown to be functionally involved in cardiovascular reactivity. Rats stressed with acute air jet-stress did not display the usual increase in blood pressure when a synaptic inhibitor was injected in the PVN [43]. Moreover, when electrically stimulated, this nucleus was shown to be involved in the regulation of sympathetic activity [44]. The PVN is also the main effector of the hormonal stress response, secreting corticotropin releasing factor that activates the hypothalamic-pituitary-adrenal (HPA) axis [45].

\section{The role of the sympathetic system}

As previously mentioned, chronic activation of the sympathetic system and/or decreased parasympathetic (vagal) is a remarkable feature of CVD [46-48]. In fact the sympathetic system contributes to endothelial dysfunction, hypertension and atherosclerosis; promotes insulin resistance and dyslipidemia; induces left ventricular hypertrophy; increases the incidence of arrhythmia; and promotes renal dysfunction by stimulating sodium and fluid retention, glomerulosclerosis and the activation of the renin-angiotensin-aldosterone system (RAAS) (for review see [49]).

The neurohormonal hypothesis of HF states that the sympathetic system is persistently activated in patients with $\mathrm{HF}$, playing a major role on its progression and establishment [50,51]. Interestingly, norepinephrine spillover, an important marker of sympathetic activity, is increased in HF, suggesting that the cause of sympathetic activation is not peripheral impaired neuronal uptake but instead an increased sympathetic neural discharge [52]. Accordingly, studies in an animal model of myocardial ischemia revealed that brain administration of beta-blockers is more effective than in the periphery [53]. Similarly, activation or blockade of NMDA receptors within the PVN (by local injection of an agonist) induced a greater change in sympathetic excitatory responses in HF rats than in sham animals (suggesting that renal sympathetic activity was elevated only in the HF condition) [54], which is in line with the finding of markedly increased expression of NMDA NR1 receptors at both mRNA and protein levels in the PVN of HF rats [54]. These data support the concept that central circuits of autonomous regulation are altered in pathological conditions. An interesting finding is the observation that B-type natriuretic peptide was firstly isolated in the brain. Whether this peptide is involved in the regulation of heart physiology through action in the central nervous system is still not known.

In a different perspective, autonomic imbalance has been also documented in mood disorders and proposed as a common mediator for mood disorders and CVD [55-57]. As an example, depressed girls display a decreased HRV, indicating increased sympathetic drive [58]. However, although evidence clearly supports the presence of autonomic cardiovascular deregulation in depressed and stressed patients, it is yet not certain whether the altered activity of the autonomic nervous system is responsible for the increased risk of mortality and medical morbidity associated with depression.

\section{The renin-angiotensin-adosterone system}

The RAAS has an important role in maintaining circulatory homeostasis. Angiotensin II stimulates aldosterone release by the adrenal cortex, leading to an increase of sodium and water reabsorption in the kidney, thus influencing blood pressure levels [59]. Therefore, it is not surprising that RAAS activation plays a central role in the development of hypertension and in the pathogenesis of HF. In fact, its pharmacological inhibition is one of the most successful strategies in the treatment of these diseases.

Although the role of aldosterone has been traditionally seen as confined to the kidney, several findings suggest that centrally activated RAAS participates in the development of hypertension and HF [60]. In fact, the intracerebroventricular infusion of aldosterone in salt-sensitive rats promoted the development of hypertension while the intracerebroventricular infusion of a mineralocorticoid receptor antagonist, like spironolactone, prevented that phenotype [61].

In addition to the effects on blood pressure, the RAAS also regulates metabolic signaling and heart rhythm. In a transgenic TG(mRen2)27 rat, which manifests increased tissue RAAS activity, renin inhibition improved systemic insulin sensitivity, insulin metabolic signaling and glucose transport [62]. Aldosterone, as well, regulates peripheral insulin resistance, which further supports its involvement in CVD (for review see [63]). Moreover, RAAS activation modulates membrane and sarcoplasmatic reticulum ion channels whose activation has proarrhythmic effects [64].

Studies in depressed patients showed that hyperaldosteronism was common among depressed patients, suggesting that increased aldosterone levels may be a mediator linking depression to unfavorable vascular events [65]. These findings suggest that RAAS action in the brain may be more important than initially thought and the better understanding of this pathway may help to comprehend how chronic stress and depression may trigger CVD.

\section{Hypothalamus-pituitary-adrenal axis}

The HPA axis is generally considered one of the principal effectors of stress response through the release of glucocorticoid hormones (GC; mainly cortisol in humans and corticosterone in rodents). These hormones contribute to restore homeostasis and to promote coping strategies to the initial insult through a series of actions both in the periphery and in the brain. GC promote gluconeogenesis, increasing the accessibility of energy to the exercising muscle; enhance cardiovascular tone; decrease feeding and appetite and optimize cognitive functions by increasing cerebral perfusion rates and local cerebral glucose utilization (for review see [66]). Moreover, through cellular receptors, namely in the $\mathrm{mPFC}$ and in the hippocampus, GC are powerful modulators of the structure and function of many regions of the CNS, exerting a powerful influence on the performance of coping behaviors in response to acute stress [67]. In spite of its beneficial effects, chronic elevation of GC contributes to a disadvantageous phenotype, namely by promoting: visceral obesity, insulin resistance and glucose intolerance, endothelial dysfunction, higher levels of low-density lipoprotein cholesterol and triglycerides, and RAAS activation (for review see [68]). In addition, together with insulin, GC stimulates the ingestion of "comfort-foods" as a compensatory hedonic mechanism [69]. This shift in caloric intake, together with elevated cortisol and insulin, increases CVD risk, showing that increased cortisol contributes for the adverse metabolic effects associated with stress $[70,71]$.

As expected, the effects of chronic stress on HPA axis tend to mimic those observed in depressed individuals, inducing elevated GC levels or altered circadian patterns, as a consequence of changes in higher control centers that blunt the negative feedback response. In animal models, chronic stress also triggers changes in brain centers that control PVN activity, namely the MPFC and the hippocampus, whose net result is an overactivation of the HPA [72].

\section{The crosstalk between the nervous and the adipose tissues}

Classic risk factors for CVD tend to coexist in the same individual, thus exponentially increasing the risk of ischemic heart disease and other adverse outcomes. The existence of such clustering suggests a common pathophysiological mechanism for all these metabolic conditions and led to the proposal, in the 80 s, of Syndrome X [more 
recently coined as metabolic syndrome (MSynd)]: "a state of insulin resistance that results in hypertriglyceridemia, low high-density lipoprotein (HDL)-cholesterol levels and hypertension" [73].

The brain is known to play a role in the development of MSynd by influencing the activity of visceral adipose tissue. In fact, sympathetic system activation, reduced serotoninergic responsivity and endocannabinoid overactivation were proposed as important neuroendocrine determinants in the pathogenesis of MSynd [74]. Among these mechanisms, the role of endocannabinoid has gained increasing interest. Briefly, the endocannabinoid system is composed of two receptors: $\mathrm{CB}-1$ and $\mathrm{CB}-2$ and two endogenous ligands: anandamide and 2-arachidonoyl-glycerol [75]. Through actions mediated by CB-1 receptors this system operates in: i) the hypothalamus, regulating energy balance by promoting appetite and motivation to eat; ii) the adipose tissue, modulating fat distribution, enhancing lipogenesis and regulating adipokines expression; iii) the liver, increasing free fatty production and insulin resistance; and iv) the muscle, decreasing glucose uptake and, thus, contributing to insulin resistance $[76,77]$. In accordance, knockout mice for $\mathrm{CB}-1$ receptors are more prone to leanness, are more resistant to diet-induced obesity and show increased central sensitivity to leptin [78]. Following this evidence, an antagonist (rimonabant) of endocannabinoid receptors, combined with an appropriated hypocaloric diet was released as a therapy to reduce weight in obese patients [79]. Rimonabant increase levels of high-density lipoproteins, decrease triglycerides and increase adiponectin. These effects were attributed not only to the central blockade of orexigenic signals mediated by CB- 1 receptors but also to its peripheral actions in different tissues [78]. Of notice here, it should be highlighted that rimonabant acts directly in the heart preventing the decrease in contractile performance of human atrial muscle mediated by endocannabinoids [80]. Thus, blockage of CB-1 receptors, both centrally and peripherally, seems to be a good approach for a wide range of patients. However the CRESCENDO trial designed to observe the effect of rimonabant in over 18,000 patients was terminated due to an increase incidence of suicide and an absence of cardiovascular benefits [81]. An option to circumvent this conundrum would be the development of a specific peripheral CB-receptor antagonist.

Although the endocannabinoid system is overactivated in MSynd, the exogenous stimuli responsible for this deregulation are still unknown. Recent data suggested that the endocannabinoid system plays a critical role in regulating activation of stress responsive systems and subsequent behavioral manifestations [82]. The deregulation of endocannabinoid signaling may originate maladaptive processes with implications over energy expenditure and food consumption. Interestingly some studies show that social stress in animals alters feeding behavior in the direction of hyperphagia [83]. Whether this is dependent on endocannabinoid signaling is still to be demonstrated.

As previously debated, the sympathetic system plays a direct role in this scenario. Chronic stressed animals submitted to a high-fat and high-sugar diet have increased neuropeptide Y release by sympathetic terminals in adipose tissue favoring the appearance of obesity and MSynd [84].

Although less extensively studied, reduced serotonergic responsivity has been proposed to play a role in the development of MSynd. The central serotonergic responsivity may be measured by the magnitude of prolactin blood level increment in response to the administration of drugs that enhance serotonergic neurotransmission. Cross-sectional studies, in community settings, showed a blunted response of prolactin to the administration of serotonergic drugs (like fenfluramine and citalopram) in MSynd and in subjects with physical inactivity [85]. These data suggest that reduced serotoninergic responsivity is involved in the pathophysiology of MSynd and in the link between depression (that is associated with serotonergic disruption) and MSynd.

Besides insulin, the adipocyte-derived hormone leptin is also a major regulator of MSynd and its importance worth being discussed. Leptin is a protein expressed mainly by adipocytes, and is involved in the regulation of food intake, energy expenditure and whole-body energy balance [86]. It is hypothesized that leptin signals the status of body energy stores to the brain (and perhaps other tissues), contributing to energy balance by decreasing food intake [87]. In obese individuals leptin levels tend to be increased, suggesting a leptin resistance phenotype. Leptin effects go well beyond energy regulation, acting directly and indirectly on heart and vessels (for review see [88]). While leptin may be regarded as cardioprotective due to its anti-apoptotic effect on myocytes, leptin excess also promotes ventricular hypertrophy and activation of inflammatory cells in the heart. At a vascular level, leptin excess promotes hypertension and atherosclerosis due to its action of the endothelium, stimulating intimal monocyte recruitment, macrophage-to-foam cell transformation, proliferation of vascular smooth muscle cells, and further secretion of pro-atherogenic cytokines [88]. Leptin resistance and hyperleptinemia are common features in obesity and MSynd and are associated with cardiovascular disease in humans.

\section{Brain activation and stress induces myocytolysis}

Until this point we have been discussing how brain injuries and chronic stress increase the risk of CVD by the deregulation of complex physiologic systems promoting hypertension, dyslipidemia, diabetes and atherosclerosis. What remains to be discussed is whether the brain-heart interaction may be more direct, by acting on the myocyte.

The most striking example of this direct interaction is the Takotsubo cardiomyopathy (or "stress cardiomyopathy") that consists of a transient left ventricular dysfunction triggered by acute emotional or physical stress, whose clinical presentation mimics AMI, with acute chest pain, transient ST-elevation and apical ballooning on echocardiography. Although its cause remains elusive, coronary artery vasospasm, coronary microcirculation dysfunction, obstruction of the left ventricular outflow tract (LVOT) and catecholamine overload have been proposed as mechanisms for the injury [89]. In spite of being initially described as a response to emotional and physical stress, recent findings demonstrated an association between stress cardiomyopathy and several CNS diseases like epilepsy [90], ischemic stroke (mainly if IC is affected) [91] and subarachnoid hemorrhage [92]. This last condition is frequently associated with troponin elevation even in the absence of cardiac history and this fact is associated with worst prognosis [93]. Whether these cardiac alterations result from stress inherent to the disease in itself or from brain stimulation is a question that remains to be clarified.

Independently of the initial insult, the histological alterations in stress cardiomyopathy consist in mild inflammatory cell infiltration, considerable increase in extracellular matrix protein levels, and contraction band necrosis (a type of cellular alteration also known as myocytolysis) [89]. Myocytolysis consists in a myofibrillar degeneration in which the cell dies in a hypercontracted state with early myofibrillar damage and anomalous irregular cross-band formations [94]. Interestingly, this kind of histopathological lesion has been related with several brain conditions not necessarily associated with Takotsubo cardiomyopathy. As revised by Samuels, this lesion is common to several neurogenic mechanisms of cardiac disease like catecholamine infusion, brain stimulation (namely the IC and hypothalamus) and stress. Interestingly, evidence shows that heart lesions may occur even in adrenalectomized animals (although less pronounced), which suggests that these findings may be dependent on the direct action of nerve terminals in the heart [94,95].

In this sense it seems that CNS may contribute to CVD in multiple manners, including direct myocyte lesion and, indirectly, due to its action in vessels, adipose tissue and hormonal milieu.

\section{The periphery modulates brain areas implicated in CVD}

As previously described, rodents submitted to CMS display alterations in autonomic parameters, like decreased HRV, suggesting 
an overactive sympathetic system that may impact on cardiovascular and metabolic functions. One would expect that once stress is over, the autonomic imbalance disappears and accompanies the improvement of the behavioral alterations seen in these animals. However, interesting data showed that recovery of depressive-like behavior in rats submitted to CMS protocol is not accompanied with a reciprocal improvement in the cardiovascular parameters [96]. Accordingly, depressed patients submitted to cognitive behavioral therapy improve mood symptoms but not autonomic function [97]. Antidepressants also seem to be ineffective in completely decreasing the exaggerated autonomic tone and high incidence of cardiovascular events observed in depressed patients (e.g. sertraline is effective in improving depression in post-myocardial infarction depressed patients but fails to decrease mortality by cardiovascular events) [98]. Therefore, although specific brain regions are involved in the initiation of cardiovascular and metabolic deregulation related to stress, other mechanisms perpetuate this cycle; probably the altered hormonal milieu (hyperleptinemia, hyperinsulinemia, hypercortisolemia and hyperaldosteronism) acts back in the brain and maintains the activation of the sympathetic system. Indeed, leptin has been widely described as a stimulus for exacerbated sympathetic drive (for review see [99]). In addition, hyperinsulinemia also promotes sympathetic activation and therefore contributes to adverse cardiovascular conditions like hypertension [100]. Aldosterone and Ang II are other mediators clearly involved in the regulation of sympathetic response by their action in the RVLM [101]. Animal studies showed that both circulating and brain Ang II promotes increased sympathetic drive leading to a hypertension-like phenotype that is reverted by the peripheral and central blockage of this system [102].

These interesting findings lead us to hypothesize (Fig. 2) that the continuous activation of the sympathetic system may be dependent on the metabolic dysfunction whose trigger is (in part) sympathetic overactivation itself, in a perpetuating feed-forward mechanism.

\section{Treatment options for stress-related CVD}

When considering all evidence and mechanisms herein revisited, some considerations are worth mentioning regarding the treatment of patients with neuropsychiatric and/or cardiovascular diseases. We herein propose a shift in the therapeutic paradigm, considering processes "from the periphery to the brain and back". In this sense, drugs that act not only in the periphery, but also in the CNS, can be of major interest. Although it is well established that chronic stress, depression and anxiety impose cardiovascular risk, it is yet to be defined what is the best strategy to pursue in these patients (whether to treat them aggressively or simply be conservative). It is of notice that some currently used drugs have both antidepressant and cardiometabolic actions. This is relevant since antidepressants were shown to be insufficient to induce a clinically meaningful normalization of the cardiovascular dysfunction associated with psychiatric conditions. Among the drugs that promote both metabolic control and recovery of depressive behavior are thiazolidinediones (or glitazones), a class of oral anti-diabetic drugs acting as sensitizers of insulin action by

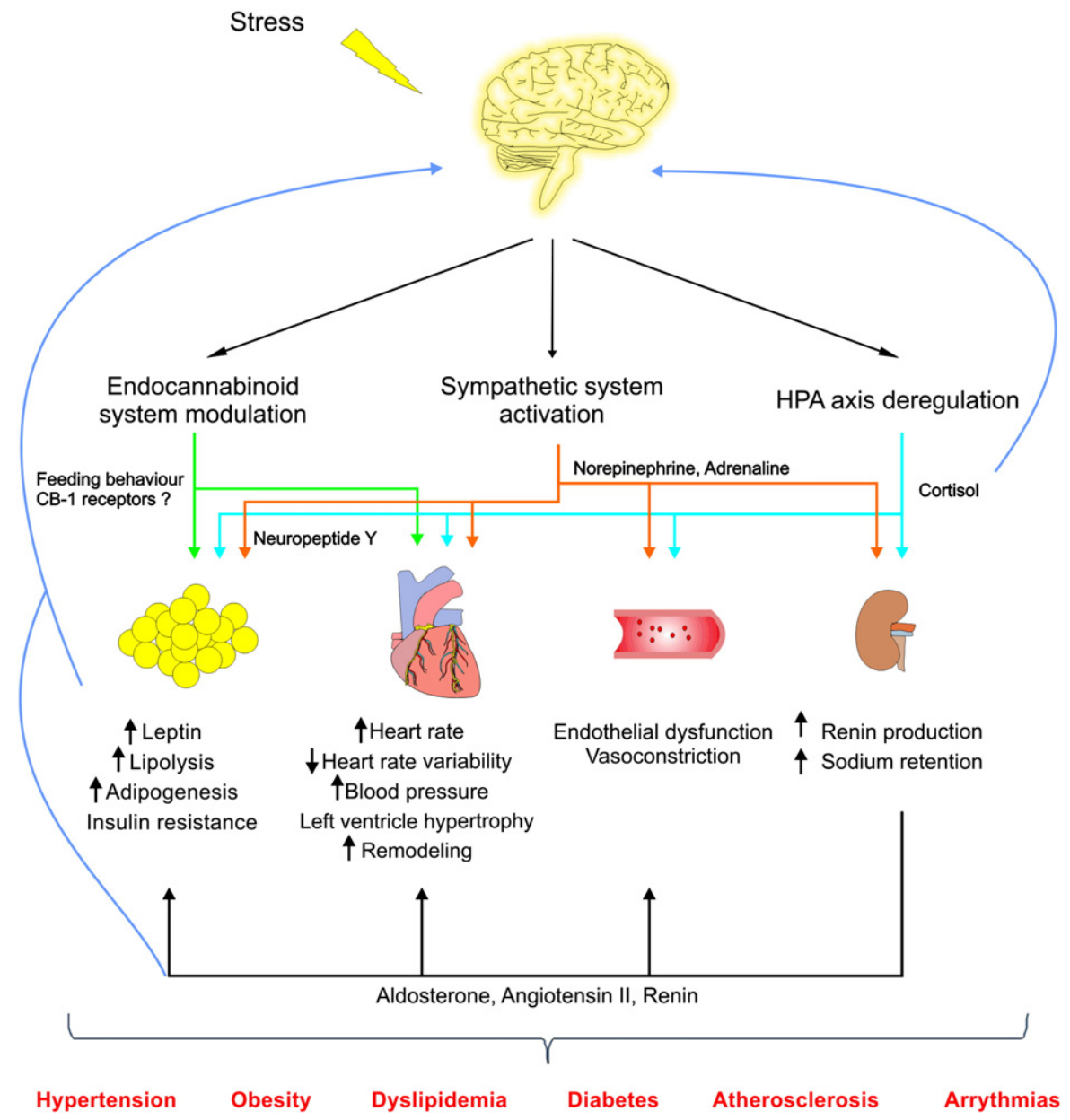

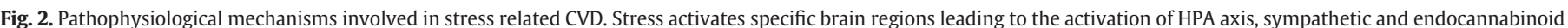



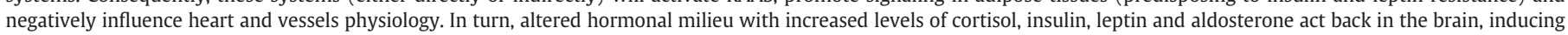
structural alterations, perpetuating this cascade. 
activating the gamma-peroxisome proliferator-activated receptors [103]. Patients treated with rosiglitazone, as a supplement to the usual antidepressant regimen, exhibited significant declines in depression severity, thus encouraging the potential use of this agent in the treatment of depressive disorders (in spite of some reported adverse effects) [104].

Instead of reducing corticosteroid levels, the aldosterone antagonist spironolactone, widely used for HF, acts by blocking its binding to mineralocorticoid receptors. This fact puts aldosterone antagonists in a good position to tackle both CVD, whose activation of the RAAS is a core feature, and stress-related disorders. In practice, in patients with HF who are taking conventional drugs, the addition of spironolactone induces a favorable sympathovagal balance [105]. However, while the beneficial effects are well documented in treating HF their use in depression may not be so encouraging, given some CNS side effects [106,107].

Beta-blockers are another class of drugs widely used in several CVD and ideally situated to bridge the brain and the cardiovascular system. Indeed, recent studies showed that beta-blockers may be safely used in depressed patients with heart disease. A recent metaanalysis concluded that addition of pindolol to an antidepressant (fluoxetine) significantly increased the percentage of patients that attained remission of depressed symptoms [108]. This suggests that beta-blockers may even be beneficial for both the behavioral and the cardiovascular consequences of depression.

As it is well known, life style modifications, such as physical exercise and diet, play an indispensible and crucial role in the management of these diseases. Physical exercise favors cardiovascular conditioning, improves functional capacity and increases HRV [109]. Exercise also has a positive effect on metabolism, diminishing glucose intolerance and insulin resistance [110]. Interestingly, the effects of physical activity are not limited to CVD but also in recovery of the depression phenotype [111]. Even though the mechanisms involved are unclear, enhanced IGF-1 production seems to be involved [110]. The promotion of physical activity should be in the mind of all physicians whether they are cardiologists, neurologists or psychiatrists.

It is expected that upcoming new information will benefit traditional treatment of CVD, most probably as a combination of both lifestyle modifications and pharmaceutical agents.

\section{Neurocardiology: final considerations}

Recent data show that highly prevalent diseases like stress-related pathology and depression, long considered as brain disorders, are increasingly found to impose a heavy burden to the cardiovascular system. Even though the heart-brain interaction is known to be bidirectional, it seems, at this point, that regulation of the CNS over heart and vessels is more prevalent as shown by the increased risk of CVD in anxious, depressed, epileptic or stroke-victims patients. The study of the regulation of cardiovascular functions (like cardiovascular reactivity) and of how they are altered by stress, CNS pathology and aging will certainly be one of the challenges of neurocardiology in the years to come. Fortunately, functional neuroimaging and neuro- and cardio-electrophysiology will help provide new insights on this matter.

From the discussion above it stands out that a better knowledge of the common mediators of CNS and cardiovascular disorders will significantly contribute to improve patient management and outcomes. Critically, this may be dependent on the construction of multidisciplinary teams and integrated approaches that direct the attention and the appropriate methodologies, for the benefit of patients.

\section{Acknowledgment}

The authors of this manuscript have certified that they comply with the Principles of Ethical Publishing in the International Journal of Cardiology [112].

\section{References}

[1] World Health Organization. The global burden of disease: 2004 update Switzerland: WHO Press; 2008.

[2] Yusuf S, Reddy S, Ounpuu S, Anand S. Global burden of cardiovascular diseases: part II: variations in cardiovascular disease by specific ethnic groups and geographic regions and prevention strategies. Circulation 2001;104:2855-64, doi:10.1161/hc4701.099488.

[3] Pozuelo L, Tesar G, Zhang J, Penn M, Franco K, Jiang W. Depression and heart disease: what do we know, and where are we headed? Cleve Clin J Med 2009;76: 59-70, doi:10.3949/ccjm.75a.08011.

[4] Frasure-Smith N, Lespérance F. Depression and cardiac risk: present status and future directions. Postgrad Med J 2010;86:193-6, doi:10.1136/hrt.2009.186957.

[5] Pratt LA, Ford DE, Crum RM, Armenian HK, Gallo JJ, Eaton WW. Depression, psychotropic medication, and risk of myocardial infarction. Prospective data from the Baltimore ECA follow-up. Circulation 1996;94:3123-9, doi: 10.1161/01.CIR.94.12.3123.

[6] Barefoot JC, Schroll M. Symptoms of depression, acute myocardial infarction, and total mortality in a community sample. Circulation 1996;93:1976, doi: 10.1161/01.CIR.93.11.1976

[7] Ariyo AA, Haan M, Tangen CM, et al. Depressive symptoms and risks of coronary heart disease and mortality in elderly Americans. Circulation 2000;102:1773, doi:10.1161/01.CIR.102.15.1773.

[8] Rugulies R. Depression as a predictor for coronary heart disease. a review and meta-analysis. Am J Prev Med 2002;23:51-61.

[9] Lesperance F. Five-year risk of cardiac mortality in relation to initial severity and one-year changes in depression symptoms after myocardial infarction. Circulation 2002:105:1049-53, doi:10.1161/hc0902.104707.

[10] Nabi H, Shipley MJ, Vahtera J, et al. Effects of depressive symptoms and coronary heart disease and their interactive associations on mortality in middle-aged adults: the Whitehall II cohort study. Heart 2010;96:1645-50, doi: 10.1136/hrt.2010.198507.

[11] Empana JP, Jouven X, Lemaitre RN, et al. Clinical depression and risk of out-ofhospital cardiac arrest. Arch Intern Med 2006;166:195-200, doi: 10.1001/archinte.166.2.195.

[12] Whang W, Kubzansky LD, Kawachi I, et al. Depression and risk of sudden cardiac death and coronary heart disease in women: results from the Nurses Health Study. J Am Coll Cardiol 2009;53:950-8, doi:10.1016/j.jacc.2008.10.060.

[13] Macchia A, Monte S, Pellegrini F, et al. Depression worsens outcomes in elderly patients with heart failure: an analysis of 48,117 patients in a community setting. Eur J Heart Fail 2008;10:714-21, doi:10.1016/j.ejheart.2008.05.011.

[14] Rutledge T, Reis VA, Linke SE, Greenberg BH, Mills PJ. Depression in heart failure a meta-analytic review of prevalence, intervention effects, and associations with clinical outcomes. J Am Coll Cardiol 2006;48:1527-37, doi 10.1016/j.jacc.2006.06.055.

[15] Laowattana S, Zeger SL, Lima JAC, Goodman SN, Wittstein IS, Oppenheimer SM Left insular stroke is associated with adverse cardiac outcome. Neurology 2006;66:477-83, doi:10.1212/01.wnl.0000202684.29640.60.

[16] Pasquini M, Laurent C, Kroumova M, et al. Insular infarcts and electrocardiographic changes at admission: results of the PRognostic of Insular CErebral infarctS Study (PRINCESS). J Neurol 2006;253:618-24, doi:10.1007/s00415-0060070-x.

[17] Tomson T, Nashef L, Ryvlin P. Sudden unexpected death in epilepsy: current knowledge and future directions. Lancet Neurol 2008;7:1021-31, doi: 10.1016/S1474-4422(08)70202-3.

[18] Nei M, Ho RT, Abou-Khalil BW, et al. EEG and ECG in sudden unexplained death in epilepsy. Epilepsia 2004;45:338-45, doi:10.1111/j.0013-9580.2004.05503.x.

[19] Kyrou I, Tsigos C. Stress hormones: physiological stress and regulation of metabolism. Curr Opin Pharmacol 2009;9:787-93, doi: 10.1016/j.coph.2009.08.007.

[20] Cannon W. Voodoo death. Am Anthropol 1942;44:169-81.

[21] Leor J, Poole WK, Kloner RA. Sudden cardiac death triggered by an earthquake. N Engl J Med 1996;334:413-9, doi:10.1056/NEJM199602153340701.

[22] Rosengren A, Hawken S, Ôunuu S, et al. Association of psychosocial risk factors with risk of acute myocardial infarction in 11119 cases and 13648 controls from 52 countries (the INTERHEART study): case-control study. Lancet 2004;364: 953-62, doi:10.1016/S0140-6736(04)17019-0.

[23] Willner P. Chronic mild stress (CMS) revisited: consistency and behaviouralneurobiological concordance in the effects of CMS. Neuropsychobiology 2005:52:90-110, doi:10.1159/000087097.

[24] Bessa JM, Mesquita AR, Oliveira M, et al. A trans-dimensional approach to the behavioral aspects of depression. Front Behav Neurosci 2009;3:1, doi 10.3389/neuro.08.001.2009.

[25] Grippo AJ, Moffitt JA, Johnson AK. Cardiovascular alterations and autonomic imbalance in an experimental model of depression. Am J Physiol Regul Integr Comp Physiol 2002;282:R1333-41, doi:10.1152/ajpregu.00614.2001.

[26] Grippo AJ, Johnson AK. Stress, depression and cardiovascular dysregulation: review of neurobiological mechanisms and the integration of research from preclinical disease models. Stress 2009;12:1-21, doi:10.1080/10253890802046281.

[27] Saper CB. Autonomic nervous system. In: Paxinos G, editor. The rat nervous system. 3rd edn. San Diego: Elsevier Academic Press; 2004. p. 761-96.

[28] Verberne AJ, Owens NC. Cortical modulation of the cardiovascular system. Prog Neurobiol 1998;54:149-68, doi:10.1016/S0301-0082(97)00056-7.

[29] Tokgozoglu SL, Batur MK, Topcuoglu MA, Saribas O, Kes S, Oto A. Effects of stroke localization on cardiac autonomic balance and sudden death. Stroke 1999;30: 1307, doi:10.1111/j.1460-9568.1996.tb00723.x. 
[30] Oppenheimer SM, Cechetto DF. Cardiac chronotropic organization of the rat insular cortex. Brain Res 1990;533:66-72, doi:10.1016/0006-8993(90)91796-J.

[31] Oppenheimer S. Cerebrogenic cardiac arrhythmias: cortical lateralization and clinical significance. Clin Auton Res 2006;16:6-11, doi:10.1007/s10286-0060276-0.

[32] Yoon BW, Morillo CA, Cechetto DF, Hachinski V. Cerebral hemispheric lateralization in cardiac autonomic control. Arch Neurol 1997;54:741-4.

[33] Barron SA, Rogovski Z, Hemli J. Autonomic consequences of cerebral hemisphere infarction. Stroke 1994;25:113-6, doi:10.1161/01.STR.25.1.113.

[34] Oppenheimer S. The anatomy and physiology of cortical mechanisms of cardiac control. Stroke 1993;24:I3-5.

[35] Cechetto DF. Identification of a cortical site for stress-induced cardiovascular dysfunction. Integr Psychol Behav Sci 1994;29:362-73, doi:10.1007/BF02691356.

[36] Cerqueira JJ, Almeida OFX, Sousa N. The stressed prefrontal cortex. Left? Right! Brain Behav Immun 2008:22:630-8, doi:10.1016/j.bbi.2008.01.005.

[37] Cerqueira JJ, Mailliet F, Almeida OFX, Jay TM, Sousa N. The prefrontal cortex as a key target of the maladaptive response to stress. J Neurosci 2007;27:2781-7, doi:10.1523/JNEUROSCI.4372-06.2007.

[38] Resstel LBM, Corrêa FM. Involvement of the medial prefrontal cortex in central cardiovascular modulation in the rat. Auton Neurosci 2006;126-127:130-8, doi: 10.1016/j.autneu.2006.02.022.

[39] Tavares RF. Corrêa FM, Resstel LBM. Opposite role of infralimbic and prelimbic cortex in the tachycardiac response evoked by acute restraint stress in rats. J Neurosci Res 2009;87:2601-7, doi:10.1002/jnr.22070.

[40] Hilz MJ, Devinsky O, Szczepanska H, Borod JC, Marthol H, Tutaj M. Right ventromedial prefrontal lesions result in paradoxical cardiovascular activation with emotional stimuli. Brain 2006;129:3343-55, doi:10.1093/brain/awl299.

[41] Allen GV, Cechetto DF. Functional and anatomical organization of cardiovascular pressor and depressor sites in the lateral hypothalamic area: I. Descending projections. J Comp Neurol 1992;315:313-32, doi:10.1002/cne.903150307.

[42] Allen GV, Cechetto DF. Functional and anatomical organization of cardiovascular pressor and depressor sites in the lateral hypothalamic area. II. Ascending projections. J Comp Neurol 1993;330:421-38, doi:10.1002/cne.903300310.

[43] Tavares RF, Pelosi GG, Corrêa FMA. The paraventricular nucleus of the hypothalamus is involved in cardiovascular responses to acute restraint stress in rats. Stress 2009;12:178-85, doi:10.1016/j.neulet.2007.08.063.

[44] Kannan H, Hayashida Y, Yamashita H. Increase in sympathetic outflow by paraventricular nucleus stimulation in awake rats. Am J Physiol 1989;256: R1325-30.

[45] Kageyama K, Suda T. Regulatory mechanisms underlying corticotropin-releasing factor gene expression in the hypothalamus. Endocr J 2009;56:335-44, doi: 10.1507/endocrj.K09E-075.

[46] Fauchier L, Babuty D, Cosnay P, Fauchier JP. Prognostic value of heart rate variability for sudden death and major arrhythmic events in patients with idiopathic dilated cardiomyopathy. J Am Coll Cardiol 1999;33:1203-7.

[47] Huikuri HV, Jokinen V, Syvänne M, et al. Heart rate variability and progression of coronary atherosclerosis. Arterioscler Thromb Vasc Biol 1999;19:1979-85, doi: 10.1161/01.ATV.19.8.1979.

[48] Joyner MJ, Charkoudian N, Wallin BG. Sympathetic nervous system and blood pressure in humans: individualized patterns of regulation and their implications. Hypertension 2010;56:10-6, doi:10.1161/HYPERTENSIONAHA.109.140186.

[49] Lambert GW, Straznicky NE, Lambert EA, Dixon JB, Schlaich MP. Sympathetic nervous activation in obesity and the metabolic syndrome: causes, consequences and therapeutic implications. Pharmacol Ther 2010:126:159-72, doi 10.1016/j.pharmthera.2010.02.002.

[50] Kaye D, Esler M. Sympathetic neuronal regulation of the heart in aging and heart failure. Cardiovasc Res 2005;66:256-64, doi:10.1016/j.cardiores.2005.02.012.

[51] Packer M. The neurohormonal hypothesis: a theory to explain the mechanism of disease progression in heart failure. J Am Coll Cardiol 1992;20:248-54.

[52] Hasking GJ, Esler MD, Jennings GL, Burton D, Johns J a, Korner PI. Norepinephrine spillover to plasma in patients with congestive heart failure: evidence of increased overall and cardiorenal sympathetic nervous activity. Circulation 1986;73:615-21, doi:10.1161/01.CIR.73.4.615.

[53] Skinner JE. Neurocardiology shows that the central, not peripheral, action of propranolol reduces mortality following acute coronary artery occlusion in the conscious pig. Integr Physiol Behav Sci 1991;26:85-97, doi: 10.1007/BF02691030

[54] Li YF, Cornish KG, Patel KP. Alteration of NMDA NR1 receptors within the paraventricular nucleus of hypothalamus in rats with heart failure. Circ Res 2003;93:990-7, doi:10.1161/01.RES.0000102865.60437.55.

[55] Carney RM, Freedland KE. Depression and heart rate variability in patients with coronary heart disease. Cleve Clin J Med 2009;76:S13-7, doi: 10.3949/ccjm.76.s2.03.

[56] Carney RM, Freedland KE, Veith RC. Depression, the autonomic nervous system, and coronary heart disease. Psychosom Med 2005:67:S29-33, doi: 10.1097/01.psy.0000162254.61556.d5

[57] Dao TK, Youssef $\mathrm{N}$ a, Gopaldas RR, et al. Autonomic cardiovascular dysregulation as a potential mechanism underlying depression and coronary artery bypass grafting surgery outcomes. J Cardiothorac Surg 2010;5:36, doi:10.1186/17498090-5-36.

[58] Tonhajzerova I, Ondrejka I, Javorka K, Turianikova Z, Farsky I, Javorka M. Cardiac autonomic regulation is impaired in girls with major depression. Prog Neuropsychopharmacol Biol Psychiatry 2010;34:613-8, doi:10.1016/j.pnpbp. 2010.02.023.

[59] Tomaschitz A, Pilz S, Ritz E, Obermayer-Pietsch B, Pieber TR. Aldosterone and arterial hypertension. Nat Rev Endocrinol 2010;6:83-93, doi: 10.1038/nrendo.2009.263.
[60] Francis J, S-guang Wei, Weiss RM, Felder RB. Brain angiotensin-converting enzyme activity and autonomic regulation in heart failure. Am J Physiol Heart Circ Physiol 2004;287:H2138-46, doi:10.1152/ajpheart.00112.2004.

[61] Huang BS, White RA, Jeng AY, Leenen FHH. Role of central nervous system aldosterone synthase and mineralocorticoid receptors in salt-induced hypertension in Dahl salt-sensitive rats. Am J Physiol Regul Integr Comp Physiol 2009;296:R994-1000, doi:10.1152/ajpregu.90903.2008.

[62] Lastra G, Habibi J, Whaley-Connell AT, et al. Direct renin inhibition improves systemic insulin resistance and skeletal muscle glucose transport in a transgenic rodent model of tissue renin overexpression. Endocrinology 2009;150:2561-8, doi:10.1210/en.2008-1391.

[63] Cooper SA, Whaley-Connell A, Habibi J, et al. Renin-angiotensin-aldosterone system and oxidative stress in cardiovascular insulin resistance. Am J Physiol Heart Circ Physiol 2007;293:H2009-23, doi:10.1152/ajpheart.00522.2007.

[64] Iravanian S, Dudley SC. The renin-angiotensin-aldosterone system (RAAS) and cardiac arrhythmias. Heart Rhythm 2008;5:S12-7, doi: 10.1016/j.hrthm.2008.02.025.

[65] Emanuele E, Geroldi D, Minoretti P, Coen E, Politi P. Increased plasma aldosterone in patients with clinical depression. Arch Med Res 2005:36:544-8, doi:10.1016/j.arcmed.2005.03.046.

[66] Sapolsky RM. How do glucocorticoids influence stress responses? Integrating permissive, suppressive, stimulatory, and preparative actions. Endocr Rev 2000;21:55-89, doi:10.1210/er.21.1.55.

[67] McEwen BS. Physiology and neurobiology of stress and adaptation: central role of the brain. Physiol Rev 2007;87:873-904, doi:10.1152/physrev.00041.2006.

[68] Whitworth JA, Williamson PM, Mangos G, Kelly JJ. Cardiovascular consequences of cortisol excess. Vasc Health Risk Manag 2005;1:291-9.

[69] Dallman MF, Pecoraro NC, Fleur SE. Chronic stress and comfort foods: selfmedication and abdominal obesity. Brain Behav Immun 2005;19:275-80, doi: 10.1016/j.bbi.2004.11.004

[70] Dallman MF, Akana SF, Pecoraro NC, Warne JP, la Fleur SE, Foster MT. Chronic stress and obesity: a new view of "comfort food". Proc Natl Acad Sci U S A 2003:100:11696-701, doi:10.1073/pnas.1934666100

[71] Reynolds RM, Labad J, Strachan MWJ, et al. Elevated fasting plasma cortisol is associated with ischemic heart disease and its risk factors in people with type 2 diabetes: the Edinburgh type 2 diabetes study. J Clin Endocrinol Metab 2010;95: 1602-8, doi:10.1210/jc.2009-2112.

[72] Raone A, Cassanelli A, Scheggi S, Rauggi R, Danielli B, De Montis MG. Hypothalamus-pituitary-adrenal modifications consequent to chronic stress exposure in an experimental model of depression in rats. Neuroscience 2007;146:1734-42, doi:10.1016/j.neuroscience.2007.03.027.

[73] Reaven GM. Banting lecture 1988. Role of insulin resistance in human disease. Diabetes 1988:37:1595-607.

[74] Fan A. Etiology of the metabolic syndrome. Curr Cardiol Rev 2007;3:232-9.

[75] Vemuri VK, Janero DR, Makriyannis A. Pharmacotherapeutic targeting of the endocannabinoid signaling system: drugs for obesity and the metabolic syndrome. Physiol Behav 2008:93:671-86, doi:10.1016/j.physbeh.2007.11.012.

[76] Cannon CP. The endocannabinoid system: a new approach to control cardiovascular disease. Clin Cornerstone 2005;7:17-26, doi:10.1016/S1098-3597(05)80064-X.

[77] Deedwania P. The endocannabinoid system and cardiometabolic risk: effects of CB1 receptor blockade on lipid metabolism. Int J Cardiol 2009;131:305-12, doi: 10.1016/j.ijcard.2008.06.033.

[78] Trillou CR, Delgorge C, Menet C, Arnone M, Soubrie P. CB1 cannabinoid receptor knockout in mice leads to leanness, resistance to diet-induced obesity and enhanced leptin sensitivity. Int J Obes 2004;28:640-8, doi: 10.1038/sj.ijo.0802583.

[79] Van Gaal LF, Rissanen AM, Scheen AJ, Ziegler O, Rössner S. Effects of the cannabinoid-1 receptor blocker rimonabant on weight reduction and cardiovascular risk factors in overweight patients: 1-year experience from the RIO-Europe study. Lancet 2005;365:1389-97, doi:10.1016/S0140-6736(05)66374-X.

[80] Bonz A, Laser M, Küllmer S, et al. Cannabinoids acting on CB1 receptors decrease contractile performance in human atrial muscle. J Cardiovasc Pharmacol 2003;41:657-64.

[81] Topol EJ, Bousser MG, Fox KA, et al. Rimonabant for prevention of cardiovascular events (CRESCENDO): a randomised, multicentre, placebo-controlled trial. Lancet 2010;14:517-23, doi:10.1016/S0140-6736(10)60935-X.

[82] Hill MN, McLaughlin RJ, Bingham B, et al. Endogenous cannabinoid signaling is essential for stress adaptation. Proc Natl Acad Sci U S A 2010;107:9406-11, doi: 10.1073/pnas.0914661107.

[83] Tamashiro KLK, Hegeman MA, Nguyen MMN, et al. Dynamic body weight and body composition changes in response to subordination stress. Physiol Behav 2007;91:440-8, doi:10.1016/j.physbeh.2007.04.004.

[84] Kuo LE, Kitlinska JB, Tilan JU, et al. Neuropeptide Y acts directly in the periphery on fat tissue and mediates stress-induced obesity and metabolic syndrome. Nat Med 2007:13:803-11, doi:10.1038/nm1611.

[85] Muldoon MF. Low central nervous system serotonergic responsivity is associated with the metabolic syndrome and physical inactivity. J Clin Endocrinol Metab 2004;89:266-71, doi:10.1210/jc.2003-031295.

[86] Rahmouni K, Haynes WG. Leptin and the cardiovascular system. Recent Prog Horm Res 2004;59:225-44

[87] Benoit SC, Clegg DJ, Seeley RJ, Woods SC. Insulin and leptin as adiposity signals. Recent Prog Horm Res 2004;59:267.

[88] Sweeney G. Cardiovascular effects of leptin. Nat Rev Cardiol 2010;7:22-9, doi: 10.1038/nrcardio.2009.224

[89] Nef HM, Möllmann H, Akashi YJ, Hamm CW. Mechanisms of stress (Takotsubo) cardiomyopathy. Nat Rev Cardiol 2010;7:187-93, doi:10.1038/nrcardio.2010.16. 
[90] Stöllberger C, Wegner C, Finsterer J. Seizure-associated Takotsubo cardiomyopathy. Epilepsia 2011;52:160-7, doi:10.1111/j.1528-1167.2011.03185.x.

[91] Yoshimura S, Toyoda K, Ohara T, et al. Takotsubo cardiomyopathy in acute ischemic stroke. Ann Neurol 2008;64:547-54, doi:10.1002/ana.21459.

[92] Das M, Gonsalves S, Saha A, Ross S, Williams G. Acute subarachnoid haemorrhage as a precipitant for Takotsubo cardiomyopathy: a case report and discussion. Int J Cardiol 2009;132:283-5, doi:10.1016/j.ijcard.2007.08.062.

[93] Miketic JK, Hravnak M, Sereika SM, Crago EA. Elevated cardiac troponin I and functional recovery and disability in patients after aneurysmal subarachnoid hemorrhage. Am J Crit Care 2010;19:522-8, doi:10.4037/ajcc2010156.

[94] Samuels MA. The brain-heart connection. Circulation 2007;116(1):77-84, doi: 10.1161/CIRCULATIONAHA.106.678995.

[95] Hawkins WE, Clower B. Myocardial damage after head trauma and simulated intracranial haemorrhage in mice: the role of the autonomic nervous system. Cardiovasc Res 1971;5:524-9, doi:10.1093/cvr/5.4.524.

[96] Grippo AJ, Beltz T, Johnson A. Behavioral and cardiovascular changes in the chronic mild stress model of depression. Physiol Behav 2003;78:703-10, doi: 10.1016/S0031-9384(03)00050-7.

[97] Carney RM, Freedland KE, Stein PK, Skala JA, Hoffman P, Jaffe AS. Change in heart rate and heart rate variability during treatment for depression in patients with coronary heart disease. Psychosom Med 2000;62:639-47.

[98] Berkman LF, Blumenthal J, Burg M, et al. Effects of treating depression and low perceived social support on clinical events after myocardial infarction: the Enhancing Recovery in Coronary Heart Disease Patients (ENRICHD) Randomized Trial. JAMA 2003;289:3106-16, doi:10.1001/jama.289.23.3106.

[99] Mark AL, Correia MLG, Rahmouni K, Haynes WG. Selective leptin resistance: a new concept in leptin physiology with cardiovascular implications. J Hypertens 2002;20:1245-50.

[100] Landsberg L. Diet, obesity and hypertension: an hypothesis involving insulin, the sympathetic nervous system, and adaptive thermogenesis. Q J Med 1986;61: 1081-90.

[101] Dupont AG, Brouwers S. Brain angiotensin peptides regulate sympathetic tone and blood pressure. J Hypertens 2010;28:1599-610, doi: 10.1097/HJH.0b013e32833af3b2.

[102] Huang BS, Ahmadi S, Ahmad M, White RA, Leenen FH. Central neuronal activation and pressor responses induced by circulating angiotensin II. Role of brain aldosterone-"ouabain" pathway. Am J Physiol Heart Circ Physiol 2010;299: H422-30, doi:10.1152/ajpheart.00256.2010.

[103] Brunton L, Lazo JS. Insulin, oral hypoglycemic agents, and the pharmacology of the endocrine pancreas. Goodman \& Gilman's The pharmacological basis of therapeutics. 11th edn. New York: McGraw-Hill Medical Pub. Division; 2006.

[104] Rasgon NL, Kenna HA, Williams KE, Powers B, Wroolie T, Schatzberg AF. Rosiglitazone add-on in treatment of depressed patients with insulin resistance: a pilot study. Sci World J 2010;10:321-8, doi:10.1100/tsw.2010.32

[105] Korkmaz ME, Müderrisoğlu H, Uluçam M, Ozin B. Effects of spironolactone on heart rate variability and left ventricular systolic function in severe ischemic heart failure. Am J Cardiol 2000;86:649-53, doi:10.1016/S0002-9149(00) 01046-8.

[106] Otte C, Hinkelmann K, Moritz S, et al. Modulation of the mineralocorticoid receptor as add-on treatment in depression: a randomized, double-blind, placebo-controlled proof-of-concept study. J Psychiatr Res 2010;44:339-46, doi:10.1016/j.jpsychires.2009.10.006.

[107] Sousa N, Cerqueira JJ, Almeida OFX. Corticosteroid receptors and neuroplasticity Brain Res Rev 2008;57:561-70, doi:10.1016/j.brainresrev.2007.06.007.

[108] Whale R, Terao T, Cowen P, Freemantle N, Geddes J. Pindolol augmentation of serotonin reuptake inhibitors for the treatment of depressive disorder: a systematic review. J Psychopharmacol 2010;24:513-20, doi: $10.1177 / 0269881108097714$.

[109] Routledge FS, Campbell TS, McFetridge-Durdle JA, Bacon SL. Improvements in heart rate variability with exercise therapy. Can J Cardiol 2010;26:303-12, doi: 10.1016/S0828-282X(10)70395-0.

[110] Duman CH, Schlesinger L, Terwilliger R, Russell DS, Newton SS, Duman RS Peripheral insulin-like growth factor-I produces antidepressant-like behavior and contributes to the effect of exercise. Behav Brain Res 2009;198:366-71, doi 10.1016/j.bbr.2008.11.016

[111] Zheng H, Liu Y, Li W, et al. Beneficial effects of exercise and its molecular mechanisms on depression in rats. Behav Brain Res 2006;168:47-55, doi: 10.1016/j.bbr.2005.10.007.

[112] Coats AJ, Shewan LG. Ethics in the authorship and publishing of scientific articles. Int J Cardiol 2011;153:239-40. 